\title{
Alterations in ruminal bacterial populations at induction and recovery from diet-induced milk fat depression in dairy cows
}

\author{
D. W. Pitta, ${ }^{* 1}$ N. Indugu, ${ }^{*}$ B. Vecchiarelli, ${ }^{*}$ D. E. Rico, $†$ and K. J. Harvatine \\ *Department of Clinical Studies, School of Veterinary Medicine, University of Pennsylvania, Kennett Square 19348 \\ †Centre de Recherche en Sciences Animales à Deschambault, Deschambault, QC, Canada, G0A 1S0 \\ ‡Department of Animal Science, The Pennsylvania State University, University Park 16802
}

\begin{abstract}
Ten ruminally cannulated Holstein cows were used in a crossover design that investigated changes in ruminal bacterial populations in response to induction and recovery from diet-induced milk fat depression (MFD). Further, the effect on the ruminal microbiota of the cows with diet-induced milk fat depression inoculated with rumen contents from non-milk fat-depressed donor cows was evaluated. Milk fat depression was induced during the first $10 \mathrm{~d}$ of each period by feeding a low-fiber, high-starch, and high-polyunsaturated fatty acid diet (26.1\% neutral detergent fiber, $28.1 \%$ starch, $5.8 \%$ total fatty acids, and $1.9 \% \mathrm{C} 18: 2$ ), resulting in a $30 \%$ decrease in milk fat yield. Induction was followed by a recovery phase, where all cows were switched to a high-fiber, low-starch, and low-polyunsaturated fatty acid diet (31.8\% neutral detergent fiber, $23 \%$ starch, $4.2 \%$ total fatty acids, and $1.2 \% \mathrm{C} 18: 2)$ and were allocated to (1) control (no inoculation) or (2) ruminal inoculation with donor cow digesta $(8 \mathrm{~kg} / \mathrm{d}$ for $6 \mathrm{~d}$ ). Ruminal samples were collected at the end of induction (d 10) and during recovery (d 13, 16, and 28), separated to solid and liquid fractions, extracted for DNA, PCR- amplified for the V1-V2 region of the $16 \mathrm{~S}$ rRNA gene, and analyzed for bacterial diversity. Results indicated that bacterial communities were different between fractions. In each fraction, differences were significant between the induction (d 10) and recovery (d 13, 16, and 28) periods; however, differences were less apparent with time during the recovery period. The MFD (d 10) was typified by a reduction in the relative sequence abundance of Bacteroidetes and an increase in the relative sequence abundance of Firmicutes and Actinobacteria across both fractions. At the genus level, relative sequence abundance of unclassified Lachnospi-
\end{abstract}

Received December 24, 2016.

Accepted August 29, 2017.

${ }^{1}$ Corresponding author: dpitta@vet.upenn.edu raceae, Butyrivibrio, Bulleidia, and Coriobacteriaceae were higher on d 10 and were positively correlated with trans-10,cis-12 CLA and the trans-10 isomer, suggesting their potential role in altered biohydrogenation reactions. A switch to the recovery diet resulted in a sharp increase in the Bacteroidetes lineages and a decrease in Firmicutes members on d 13; however, this shift appears to stabilize by d 28 , indicating the restoration process for ruminal bacteria from an altered state is gradual and complex. Inoculation of $10 \%$ of rumen contents from non-MFD donor cows to MFD cows revealed this procedure had transient effects on only a few bacterial populations, and such effects disappeared after d 16 following cessation of inoculation. It can be concluded that alterations in milk FA profiles at induction are preceded by microbial alterations in the rumen driven by dietary changes.

Key words: biohydrogenation, milk fat depression, ruminal microbiome, fatty acid isomers

\section{INTRODUCTION}

Diet-induced milk fat depression (MFD) in dairy cows is characterized by a specific reduction in milk fat yield in response to bioactive fatty acids produced in the rumen when feeding rapidly fermentable diets that are high in PUFA (Bauman and Griinari, 2003). Milk fat depression is a classic example of the interactions between dietary nutrients, the gastrointestinal microbiota and tissue physiology (Rico et al., 2015a). In ruminants, dietary PUFA are converted to saturated fats by microbes through biohydrogenation $(\mathbf{B H})$ in the rumen. It has been reported during the course of diet-induced MFD that rumen fermentation is altered, causing a shift in BH pathways, resulting in specific trans FA that inhibit milk fat synthesis in the mammary gland, leading to MFD (Harvatine et al., 2009). The severity of MFD is dependent upon the rate and extent of $\mathrm{BH}$ and the specific intermediates formed in the rumen, which are influenced by the concentrations of PUFA and fermentable carbohydrates in the diet 
(Jenkins et al., 2008; Fuentes et al., 2009). Therefore, MFD in dairy cows is the direct consequence of rumen microbial activity that is responsive to diet. However, the interaction between dietary factors and the rumen microbiota and their gene repertoire leading to MFD is not well understood.

Rico and Harvatine (2013) and Rico et al. (2014) have described models for induction and recovery from classic diet-induced MFD. By feeding a low-fiber, highstarch, high-PUFA diet (26.1\% NDF, 28.1\% starch, $5.8 \%$ total fatty acids, and $1.9 \% \mathrm{C} 18: 2)$ to lactating dairy cows, those authors induced MFD and noted a reduction by $30 \%$ in milk fat yield by $10 \mathrm{~d}$. After establishment of MFD, cows were switched to a high-fiber, low-starch, low-PUFA diet (31.8\% NDF, 23\% starch, $4.2 \%$ total fatty acids, and $1.2 \% \mathrm{C} 18: 2)$ and recovery from MFD was achieved in $15 \mathrm{~d}$. In response to these dietary changes, a 2-phase adaptation of milk FA profiles was reported during both induction and recovery periods. During the induction phase, cis-9,trans-11 CLA peaked on d 3 and progressively reduced, whereas trans-10,cis-12 CLA progressively increased during the induction period. However, in the recovery period, cis-9,trans-11 CLA initially increased and returned to baseline and trans-10,cis-12 CLA gradually decreased. Rico et al. (2014) concluded that the time course for induction and recovery from MFD was dependent on ruminal adaptation more than the dietary substrate available for BH. Nonetheless, how microbes respond to these dietary changes and which microbes are associated with different CLA isomers are not known.

The rumen microbiota is composed of bacteria, protozoa, fungi, and archaea that live in a symbiotic relationship and are collectively responsible for microbial fermentation in the rumen (Morgavi et al., 2013). Evidence indicates that changes in dietary components such as NDF, CP, and starch can significantly alter the composition of the rumen microbial communities (Pitta et al., 2010, 2014a,c,d). Whereas dietary structural carbohydrates and NFC and proteins are needed for microbial growth in the rumen, supplementation of fatty acids (FA) is not necessary, as microbes can synthesize their own FA (Garton, 1977). Elevated concentrations of PUFA are considered toxic to rumen microbes because PUFA form complexes with the cell walls of rumen microbes and interfere with rumen fermentation (Kim et al., 2002). Higher concentrations of PUFA and starch in the diet can alter the $\mathrm{BH}$ pathways leading to MFD (Rico and Harvatine, 2013). However, a paucity of information exists on the role of microbes in $\mathrm{BH}$ and the mechanism of action leading to MFD. Utilizing the induction-recovery model of diet-induced MFD (Rico et al., 2014), the current study investigated the changes in bacterial populations based on their relative sequence abundance at induction and their change as dairy cows recovered from MFD, with dietary intervention alone and dietary intervention combined with inoculation from non-MFD cows.

\section{MATERIALS AND METHODS}

The recovery ruminal samples used for bacterial community analysis in our study were collected and archived from a recently published report (Rico et al., 2014) that investigated production responses and included FA profiles during induction and recovery from MFD. Experimental details were previously described by Rico et al. (2014) and were approved by the Pennsylvania State University Institutional Animal Care and Use Committee. Briefly, 10 ruminally cannulated Holstein cows were randomly assigned to treatment sequences in a crossover design with 28 -d periods. Two additional cows fed a high-fiber, low-starch, low-PUFA diet $(31.8 \%$ NDF, $23 \%$ starch, $4.2 \%$ total FA, and 1.2\% C18:2; Supplemental Table S1; https://doi.org/ 10.3168/jds.2016-12514) were used as rumen digesta donors. Each treatment period included a 10-d MFD induction and an 18-d MFD recovery phase. During the MFD induction phase, the 10 treatment cows were fed a low-fiber, high-starch, high-PUFA diet $(26.1 \%$ NDF, $28.1 \%$ starch, $5.8 \%$ total FA, and $1.9 \%$ C18:2; Supplemental Table S1). During the subsequent MFD recovery phase, the cows were fed a high-fiber, lowstarch, low-PUFA diet (31.8\% NDF, 23\% starch, $4.2 \%$ total FA, and 1.2\% C18:2; Supplemental Table S1) with or without ruminal digesta inoculation. Rumen digesta inoculation of the 5 treated cows included removal of $8 \mathrm{~kg}$ of whole-rumen digesta at $1300 \mathrm{~h}$ on d 11 to 16 (recovery period) and replacement with digesta from the non-MFD donor cows. On each day of inoculation, rumen digesta was collected from 5 different locations of the rumen (cranial dorsal, cranial ventral, central, caudal dorsal, and caudal ventral) in both donor animals, mixed, and about $8 \mathrm{~kg}$ was transferred to each recipient. Rumen samples (approximately $300 \mathrm{~g}$ ) were collected from the 10 experimental cows on d 13, 16, and 28 , strained through $0.5-\mathrm{mm}$ mesh, and solid and liquid fractions were frozen and stored at $-80^{\circ} \mathrm{C}$. The same procedure was carried out to archive subsamples of the mixed donor digesta samples.

\section{DNA Extraction and PCR}

The rumen samples were processed for genomic DNA using a modified version of the PSP Spin Stool DNA Plus Kit (Invitek, Berlin, Germany) as per the method 
described by Dollive et al. (2012). Approximately 300 $\mathrm{mg}$ of sample was placed in a Lysing Matrix E tube (MP Biomedicals, Solon, $\mathrm{OH}$ ) and bead beaten in 1,400 $\mu \mathrm{L}$ of stool stabilizer from the PSP kit with a Mini-Beadbeater 16 (BioSpec, Bartlesville, OK) to break open cell walls and release nucleic acid material. Samples were then heated at $95^{\circ} \mathrm{C}$ for $15 \mathrm{~min}$, placed on ice for $1 \mathrm{~min}$, and centrifuged at room temperature at 13,400 $\times g$ for 1 min. The supernatant was then transferred to the PSP InviAdsorb tubes (BioSpec) and the rest of the protocol for the PSP Spin Stool DNA Plus was followed according to the manufacturer's instructions (http:// www.molecular.stratec.com/files/inhalte/Manuals/ PSPSpinStool_StoolPlusKit\%5b1\%5d.pdf). The extracted DNA was amplified using the bacterial-specific primers (BSF8 = 5'-GAGTTTGATCCTGGCTCAG-3'; BSR357 = 5'-CTGCTGCCTYCCGTA-3'), annealing to the V1-V2 region of the $16 \mathrm{~S}$ rRNA bacterial gene as described in McKenna et al. (2008). The V1-V2 region was recommended for metagenomic surveys of bacterial diversity studies (Wu et al., 2010; Salipante et al., 2013); hence, this region was used in the current study. Further, the forward primer carried the Ion Torrent trP1 (5'-CCTCTCTATGGGCAGTCGGTGAT-3') and the reverse primer carried the A adapter (5'-CCATCTCATCCCTGCGTGTCTCCGACTCAG- ${ }^{\prime}$ ), followed by a 10 to 12 nucleotide sample-specific barcode sequence and GAT barcode adapter as described in Indugu et al. (2016). Polymerase chain reaction was performed using the Accuprime Taq DNA polymerase System (Invitrogen, Carlsbad, CA). The thermal cycling conditions involved an initial denaturing step at $95^{\circ} \mathrm{C}$ for 5 min followed by 25 cycles (denaturing at $95^{\circ} \mathrm{C}$ for $30 \mathrm{~s}$, annealing at $56^{\circ} \mathrm{C}$ for $30 \mathrm{~s}$, extension at $72^{\circ} \mathrm{C}$ for $90 \mathrm{~s}$ ) and, finally, an extension step at $72^{\circ} \mathrm{C}$ for 8 min. The amplicons from each DNA sample, which were amplified in triplicate, were pooled and bead purified using Beckman Coulter Agencourt AMPure XP Beads (Beckman Coulter, Brea, CA). The amplicons generated for each sample were pooled in equimolar concentration and subjected to pyrosequencing on an Ion Torrent (Thermo Fisher Scientific, Carlsbad, CA) PGM sequencer.

\section{Sequencing, Data Analysis, and Statistical Analysis}

The $16 \mathrm{~S}$ pyrosequence reads were analyzed using the Quantitative Insights Into Microbial Ecology (QIIME) bioinformatics pipeline, version 1.8.0 (Caporaso et al., 2010a). Reads were discarded if they did not match the expected sample-specific barcode and $16 \mathrm{~S}$ primer sequences (forward and reverse primers), or if they were shorter than $50 \mathrm{bp}$ or longer than $480 \mathrm{bp}$, or if they contained 1 or more ambiguous base calls. Reads were also discarded if a long homopolymer sequence was present; the threshold used was $5 \mathrm{bp}$.

Operational taxonomic units (OTU) were formed at 97\% similarity using UCLUST (Edgar, 2010). Taxonomic assignments within the GreenGenes taxonomy (12/10 release; McDonald et al., 2012) were generated using the RDP Classifier version 2.2 (Wang et al., 2007). We randomly subsampled (rarified) the resulting OTU to 1,531 sequences per sample. Representative sequences for each OTU were aligned with PyNast (Caporaso et al., 2010b). The resultant multiple sequence alignment was used to infer a phylogenetic tree with FastTree (Price et al., 2010).

Two measures of $\alpha$ diversity were calculated: Shannon entropy, an indicator of evenness in community structure, and richness, the number of OTU observed. The principal coordinates analysis was performed on pairwise unweighted UniFrac distances (Lozupone and Knight, 2005).

The measured $\alpha$ diversity matrices were compared between the groups using Wilcoxon rank and KruskalWallis rank sum test for treatment and day, respectively. A nonparametric permutational multivariate ANOVA test (Anderson, 2001), implemented in the vegan package for R (version 3.1.1; R Core Team, 2014), was used to test the effects of day of sampling and treatment within the day of sampling on overall community composition, as measured by weighted UniFrac distance. To test for differences in taxa abundance, only taxa appearing in at least $75 \%$ of the samples were considered. The raw read counts from the $16 \mathrm{~S}$ OTU abundance table were collapsed at taxonomic rank and compositionally normalized such that each sample sums to 1 .

The relative sequence abundance (referred to as relative abundance from this point forward in the text) of the bacterial populations at the respective taxonomic rank were analyzed using the MIXED procedure of SAS with repeated measures (version 9.3, SAS Institute Inc., Cary, NC), as described by Rico et al. (2014). Briefly, the model included the random effect of treatment sequence, period, and cow nested in treatment sequence and the fixed effect of treatment, time, the interaction of treatment and time, and the last day of the induction period (d 10) as a covariate. Time was the repeated variable and cow by period was the subject. The covariance structures such as $\mathrm{ARH}(1), \operatorname{AR}(1)$, or CS based on Akaike information criteria were used. The Kenward-Roger denominator degree of freedom adjustment was used. Pearson correlation coefficients were calculated between production measures from Rico et al. (2014) and relative abundance data of bacterial taxa using the standard $\mathrm{R}$ function cor.test. 


\section{RESULTS}

\section{Sequencing Details}

Approximately 722,562 reads were analyzed from 156 bacterial communities. One sample consisting of fewer than 200 reads was removed from the analysis. All the remaining samples had an average 4,249 sequences. Approximately 49,160 OTU were identified by clustering at $97 \%$ sequence similarity. Representative sequences from the OTU were assigned to 27 bacterial phyla and 179 genera; however, only 30 genera were present across $75 \%$ of samples.

\section{Species Richness and Diversity}

The number of estimated bacterial species (OTU) is represented by species richness in both the liquid and solid fractions (Figure 1A and $1 \mathrm{C}$ ), and distribution of OTU across samples was represented by Shannon diversity index (Figure 1B and 1D). Species richness and diversity was lower $(P<0.05$; Kruskal Wallis rank sum test) on d 10 compared with all days of the recovery period across both fractions. We found no evident differences between inoculated and control treatments except for d $16(P<0.05$; Wilcoxon rank sum test $)$ in the liquid fraction, where the inoculated treatment showed greater values for both species richness and diversity (Figure 1A and 1B; Supplemental Table S2; https://doi.org/10.3168/jds.2016-12514).

\section{Community Comparison}

Weighted UniFrac distances were calculated based on relative abundance information of OTU in bacterial communities. These distance matrices were visualized using principal coordinate analysis (Figure 2). Grouping of bacterial communities was different $(P<0.01$; Supplemental Table S3; https://doi.org/10.3168/jds .2016-12514) between fractions. In each fraction, bacterial communities on d 10 were significantly different $(P$ $<0.01$ ) to that of d 13,16 , and 28 bacterial communities in the recovery period. However, the magnitude of difference gradually declined from d 13 to 28 , as noted by the $P$-values (Table 1 ). Differences in bacterial communities were much more apparent in the liquid when compared with the solid fraction. No differences were noted between controls and inoculated bacterial communities, except for a trend on d 16 in the liquid fraction. Notably, bacterial communities in the donor inoculum clustered with those of 28-d samples in both fractions (Supplemental Figure S1; https://doi.org/10 $.3168 /$ jds.2016-12514).

\section{Taxonomic Composition of Bacterial Communities}

About 27 different bacterial phyla were identified in this study; however, only 9 phyla were identified across $75 \%$ of the samples (Tables 2 and 3; Supplemental Figures S2 and S3; https://doi.org/10.3168/jds .2016-12514). Firmicutes and Bacteroidetes together constituted 85 to $86 \%$ of the total bacterial sequences across both fractions. In the liquid fraction, among the Bacteroidetes phylum, genera with the most relative abundance were unclassified Bacteroidales (11.0\%), Prevotella (10.6\%), and S24-7 (3.5\%) in the liquid fraction (Table 2). Prevotella was the most dominant (24.3\%), followed by unclassified Bacteroidales (9.0\%) and $524-7(5.2 \%)$ in the solid fraction (Table 3).

Among the Firmicutes phylum, the relative abundance of the most dominant genera were unclassified Clostridiales (15.5\%), unclassified Lachnospiraceae $(11.2 \%)$, unclassified Ruminococcaceae (11.1\%), Ruminococcus (6.0\%), Butyrivibrio (3.2\%), unclassified Mogibacteriaceae (2.4\%), and Bulleidia (1.6\%) in the liquid fraction (Table 2). In the solid fraction (Table 3 ), the relative abundance of unclassified Clostridiales (14.5\%), unclassified Lachnospiraceae (14.0\%), unclassified Ruminococcaceae (5.5\%), Butyrivibrio (3.3\%), and Bulleidia (0.8\%) were found to be the most dominant genera.

Actinobacteria, Cyanobacteria, Fibrobacteres, Proteobacteria, Spirochaetes, Tenericutes, and TM7 were among the phyla whose relative abundance was less than 1\%. Genera representing these phyla and Bacteroidetes and Firmicutes that were present in $75 \%$ of the communities were considered for analysis (Tables 2 and 3). Actinobacteria, Fibrobacteres, Spirochaetes, and TM7 were represented by unclassified Coriobacteriaceae, Fibrobacter, Treponema, and F16 genera respectively in both fractions.

\section{Bacterial Populations Associated with MFD}

Although the same bacterial taxa were present in the rumen of all experimental and donor dairy cows, significant differences were noted in the relative abundance of respective bacterial taxa at induction and during the recovery period in both fractions. Day 10 was marked by a significantly higher $(P<0.01)$ relative abundance of Firmicutes and Actinobacteria and a lower abundance of Bacteroidetes compared with the recovery period across both fractions (Tables 2 and 3; Supplemental Table S4 and Supplemental Figures S2 and S3; https://doi.org/10.3168/jds.2016-12514); these changes were more prominent in the liquid compared with the solid fraction. Among the Bacteroidetes members, most 
genera were observed to be lower $(P<0.01)$ in relative abundance in the liquid fraction (Table 2; Supplemental Table S5 and Supplemental Figure S4; https://doi .org/10.3168/jds.2016-12514), whereas only the most dominant genera such as unclassified Bacteroidales and Prevotella were lower $(P<0.05)$ in the solid fraction
(Table 3; Supplemental Table S5 and Supplemental Figure S5; https://doi.org/10.3168/jds.2016-12514).

Firmicutes had the highest relative abundance, accounting for approximately $57 \%$ in the liquid and $46 \%$ in the solid fraction on d 10 (Tables 2 and 3; Supplemental Figures S2 and S3; https://doi.org/10.3168/

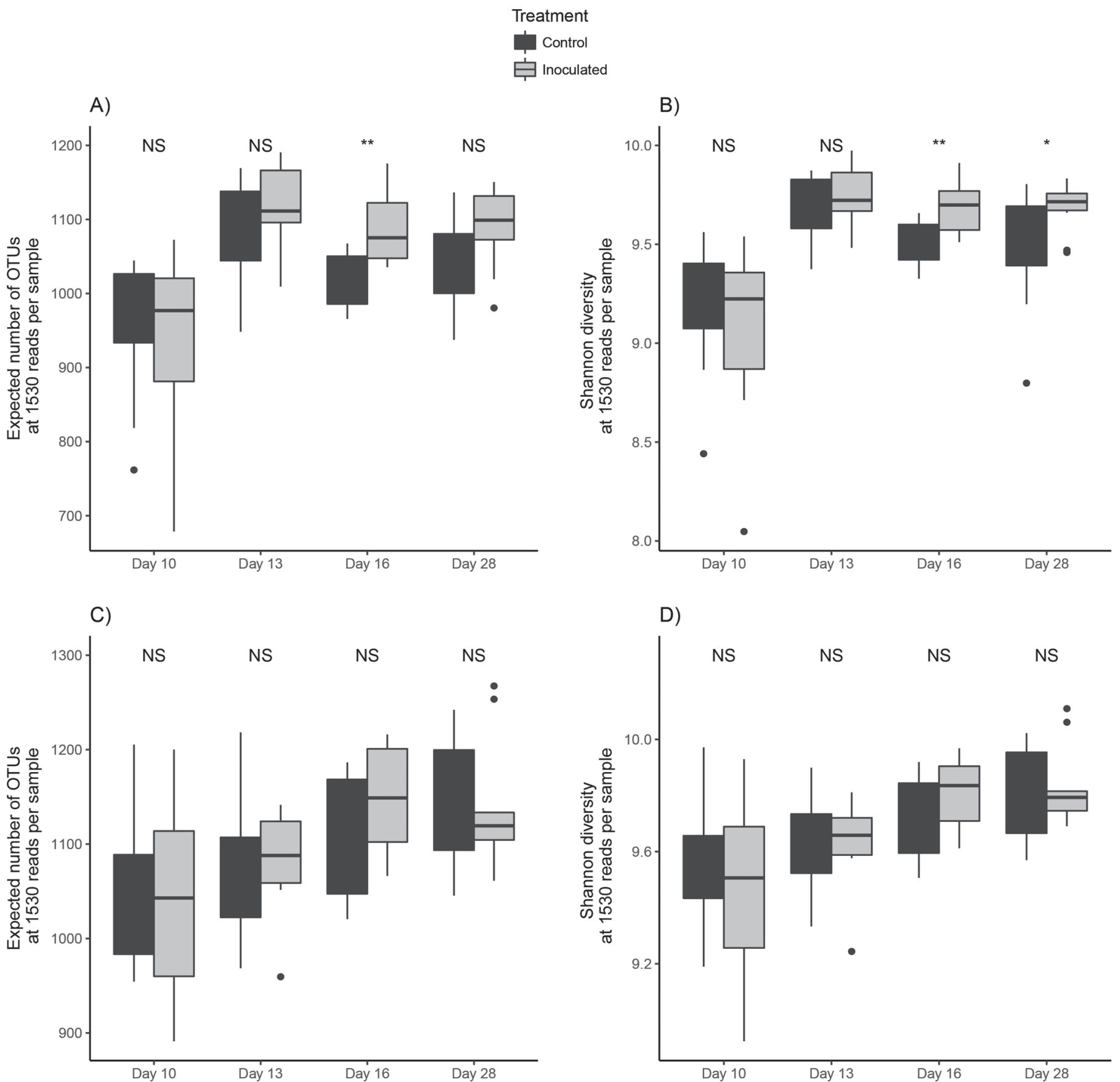

Figure 1. Measurement of community diversity ( $\alpha$ diversity) of the rumen bacterial communities by the (A) number of observed species between days in the liquid fraction, (B) Shannon diversity between days in the liquid fraction, (C) number of observed species between days in the solid fraction, and (D) Shannon diversity between days in the solid fraction $\left({ }^{*} P<0.05,{ }^{*} P<0.01\right)$ between treatments. OTU $=$ operational taxonomic unit. The box plots show the range of values, $25 \%$ (lower whisker), $50 \%$ (middle box), and 25\% (upper whisker) quartiles, the median (horizontal line), and outliers (dots). 
jds.2016-12514). The ratio between Bacteroidetes and Firmicutes was much lower $(P<0.01)$ on d 10 when compared with other days of sampling in both fractions (Figure 3a and b), possibly indicating a disruption in the bacterial populations. In the liquid fraction, several genera of Firmicutes, including unclassified Lachnospiraceae, unclassified Ruminococcaceae, Butyrivibrio, Bulleidia, and Mogibacterium, were higher in relative abundance $(P<0.01)$ on d 10 compared with the recovery period (Table 2; Supplemental Table S5 and Supplemental Figure S4; https://doi.org/10.3168/jds .2016-12514). However, in the solid fraction, only unclassified Lachnospiraceae, Butyrivibrio, and Bulleidia were found to be significantly higher in relative abundance (Table 3; Supplemental Table S6 and Supplemental Figure S5; https://doi.org/10.3168/jds.2016 -12514). In contrast, genus Ruminococcus was lower on d 10 in both fractions.

Fibrobacter was lower $(P<0.01)$ in the solid fraction, whereas Treponema was lower in relative abundance $(P$ $<0.01)$ in the liquid fraction on d 10 compared with the recovery period. Family F16 was lower on d 10 when compared with d-28 samples in both fractions. (Tables 2 and 3; Supplemental Tables S5 and S6 and Supplemental Figures S2 and S3; https://doi.org/10 $.3168 /$ jds.2016-12514)

\section{Bacterial Dynamics During the Recovery Period}

Following the switch from a low-NDF, high-starch, high-PUFA diet to a high-NDF, low-starch, low-PUFA diet, changes in the rumen bacterial populations became evident as early as d 13. A spike in the relative abundance of Bacteroidetes was noted in both fractions (Tables 2 and 3; Supplemental Figures S2 and S3, https://doi.org/10.3168/jds.2016-12514). Again, this

Table 1. Permutational multivariate ANOVA for effect of day and effect of treatment within day on ruminal bacterial community composition

\begin{tabular}{lccccc}
\hline & \multicolumn{2}{c}{ Fluid } & & \multicolumn{2}{c}{ Solid } \\
\cline { 2 - 3 } \cline { 5 - 6 } Item & $\mathrm{R}^{2}$ & $P$-value & & $\mathrm{R}^{2}$ & $P$-value \\
\hline Day & 0.29 & 0.001 & & 0.17 & 0.001 \\
10 vs. 13 & 0.30 & 0.001 & & 0.16 & 0.001 \\
10 vs. 16 & 0.27 & 0.001 & & 0.10 & 0.006 \\
10 vs. 28 & 0.31 & 0.001 & & 0.21 & 0.001 \\
13 vs. 16 & 0.08 & 0.003 & & 0.08 & 0.020 \\
13 vs. 28 & 0.10 & 0.002 & & 0.08 & 0.007 \\
16 vs. 28 & 0.05 & 0.058 & & 0.06 & 0.084 \\
Treatment & 0.01 & 0.371 & & 0.01 & 0.860 \\
Treatment: Day & 0.02 & 0.772 & & 0.03 & 0.709 \\
Treatment on d 10 & 0.03 & 0.813 & & 0.05 & 0.470 \\
Treatment on d 13 & 0.04 & 0.625 & & 0.04 & 0.732 \\
Treatment on d 16 & 0.08 & 0.113 & & 0.04 & 0.579 \\
Treatment on d 28 & 0.04 & 0.496 & & 0.03 & 0.893 \\
\hline
\end{tabular}
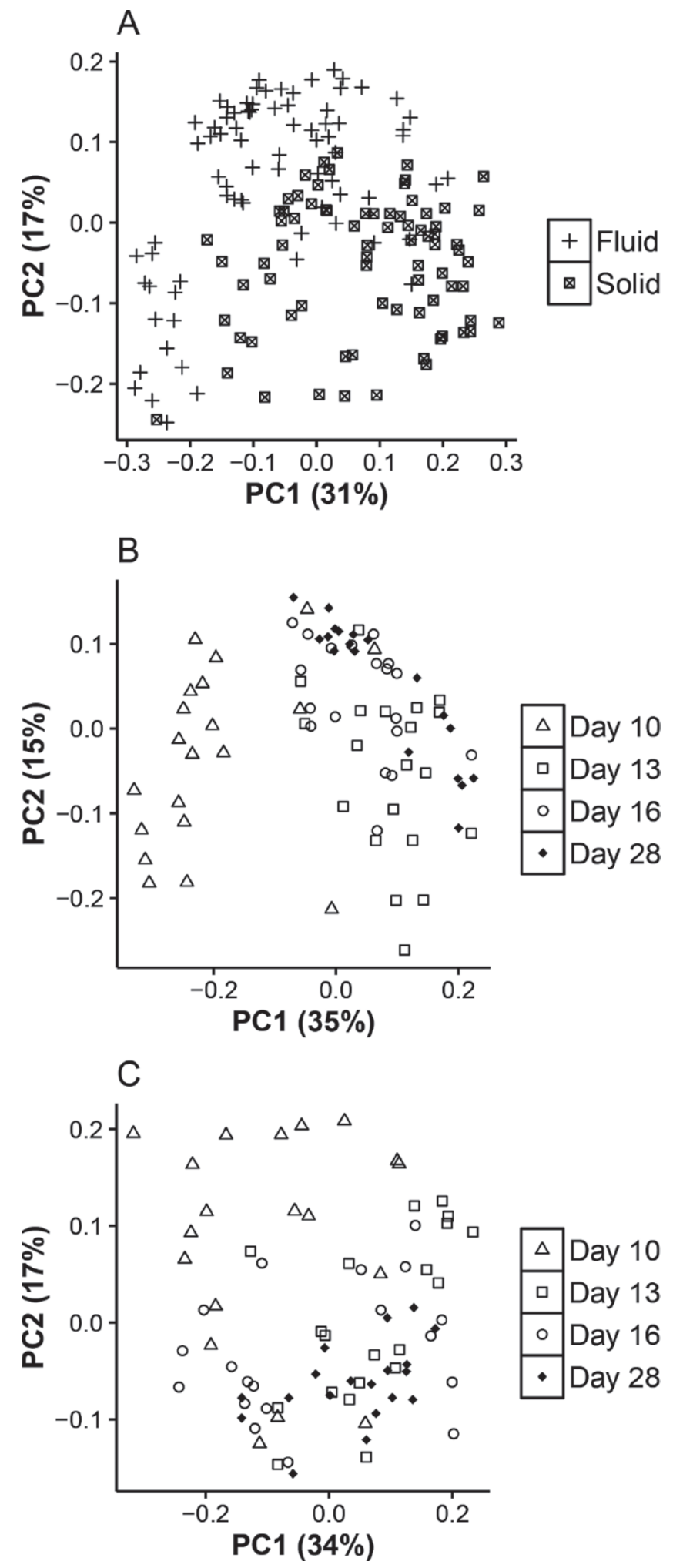

Figure 2. Comparison of bacterial community composition (A) between the fractions (solid and liquid), (B) between days (samples from d 10, 13, 16, and 28) in the liquid fraction, and (C) between days (samples from d 10,13,16, and 28) in the solid fraction. The principal coordinate $(\mathrm{PC})$ plots show weighted pairwise UniFrac distances between samples. 


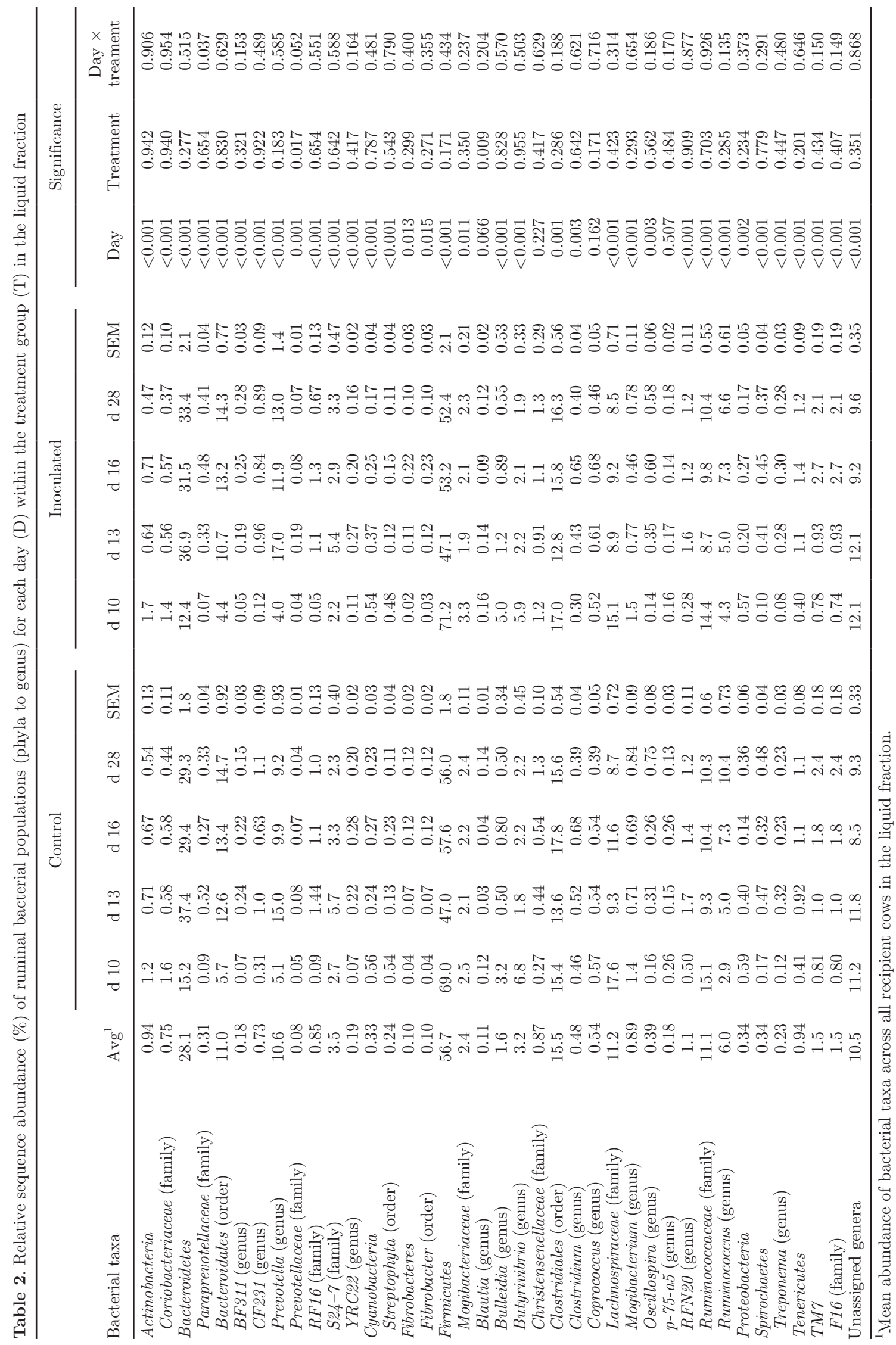









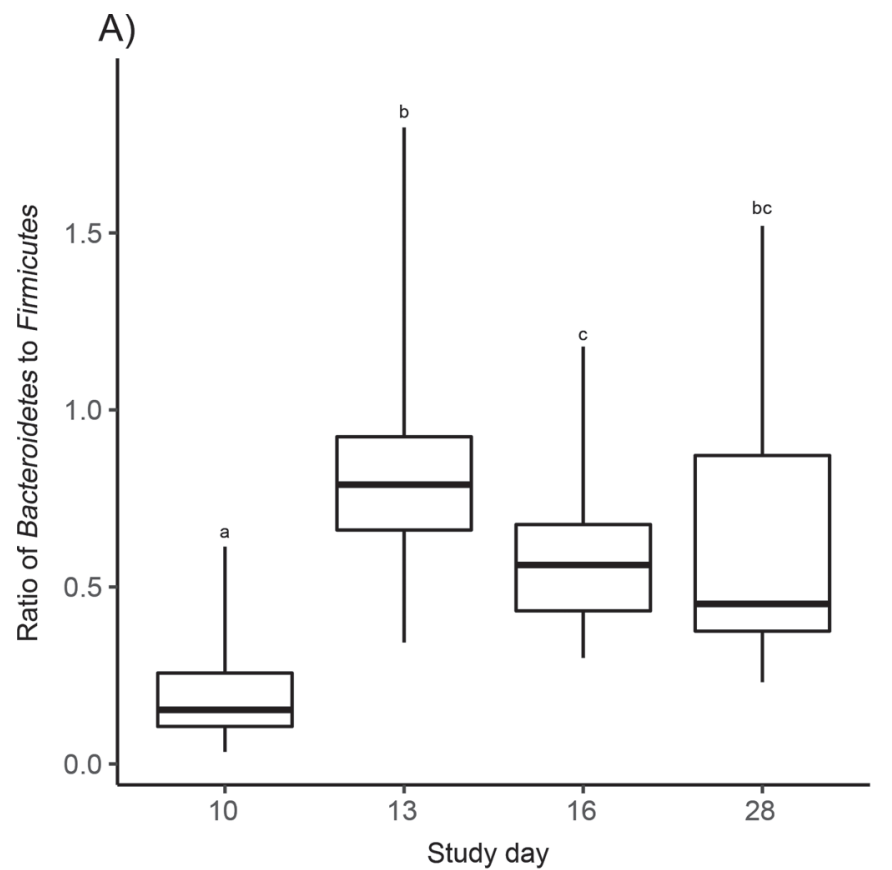

B)

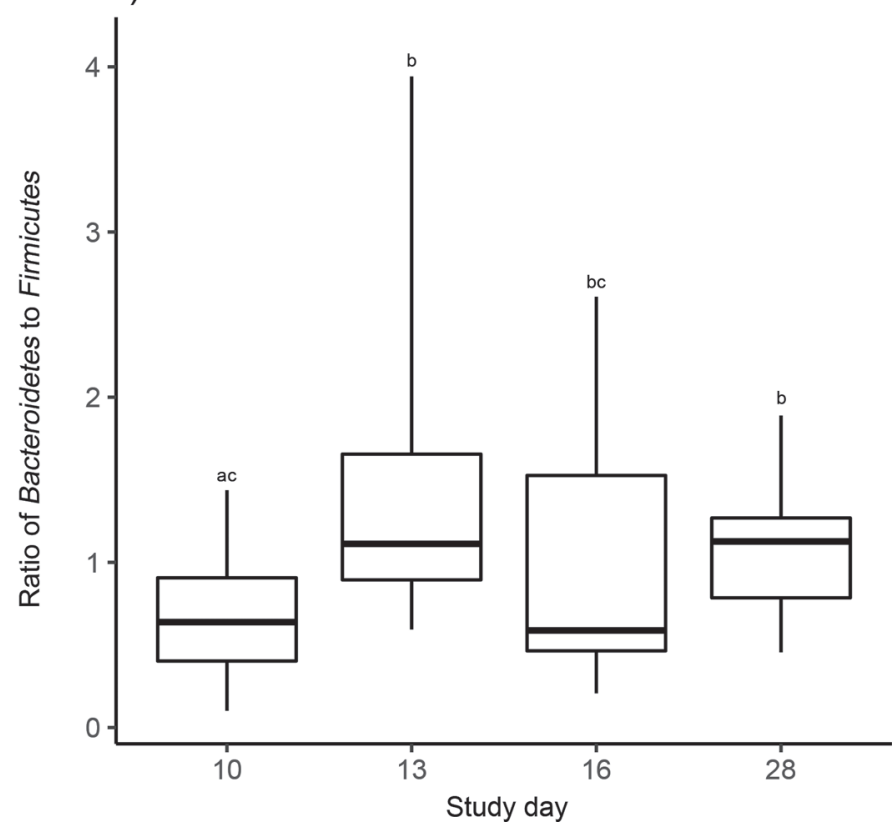

Figure 3. Ratio of phyla Bacteroidetes to Firmicutes at induction and during the recovery period for the (A) liquid and (B) solid fraction. The box plots show the range of values, $25 \%$ (lower whisker), $50 \%$ (middle box), and 25\% (upper whisker) quartiles, the median (horizontal line), and outliers (dots). Different letters indicate significant differences between study day (Wilcoxon test, $P<0.05$ ).

increase in Bacteroidetes was more evident in the liquid fraction compared with the solid fraction. In contrast, the contribution from Firmicutes was reduced. However, these changes in Bacteroidetes and Firmicutes did not persist on d 16 and 28. The relative abundance of Proteobacteria and Actinobacteria decreased in both fractions from d 10 to 28, whereas Tenericutes and TMY increased in both fractions. Spirochaetes increased in only the liquid fraction (Tables 2 and 3, Supplemental Figures S2 and S3).

In the liquid fraction, the relative abundance of the majority of genera in the Bacteroidetes phylum increased $(P<0.01)$ by 2 to 3 fold on $d 13$ of the recovery diet and then stabilized by d 28 . However, only a few lineages, such as Prevotella and S24-7, showed differences in both fractions. No significant differences were noted between d 16 and 28. Most genera in Firmicutes numerically decreased from d 13 to 28; however, significant differences were observed for Clostridiales and Mogibacterium in both fractions (Tables 2 and 3; Supplemental Tables S5 and S6; https://doi.org/10.3168/ jds.2016-12514).

\section{Effect of Rumen Content Inoculation on Resident Bacterial Populations}

Overall, the effects of inoculation on the rumen bacterial populations were insignificant compared with the effects of sampling time (Supplemental Tables S7 and S8; https://doi.org/10.3168/jds.2016-12514). The influence of inoculation on rumen microbiota began to emerge on d 13, slightly increased on d 16, but disappeared by d 28. Particularly, the number of observed species and Shannon diversity were higher for the inoculated group on d 16 and diminished by d 28 (Figure 1). These changes were more noticeable in the liquid fraction compared with the solid fraction. Very few genera showed a treatment difference and these differences were evident predominantly on d 16 in both fractions (Supplemental Table S8).

\section{Associations Between Production Responses and Bacterial Taxa}

In the liquid fraction, the relative abundance of genera from the Bacteroidetes phylum showed no positive correlations with milk yield, protein, or fat concentrations; however, a few genera, such as Bacteroidales, CF231, and $R F 16$, showed negative correlation with milk yield (Table 4). Notably, most of these genera showed negative correlations with all FA isomers. In contrast, in the Firmicutes phylum, the relative abundance of Lachnospiraceae and Butyrivibrio showed positive correlations with milk yield and protein, but not with total fat concentrations in milk. These 2 genera also showed positive correlations with cis-9,trans-11 CLA, trans-10,cis-12 CLA, and the trans-10 isomer. Interestingly, Coriobacteriaceae showed negative correlation with total fat and 
Table 4. Association between relative sequence abundance of bacterial taxa and production responses in the liquid fraction ${ }^{1}$

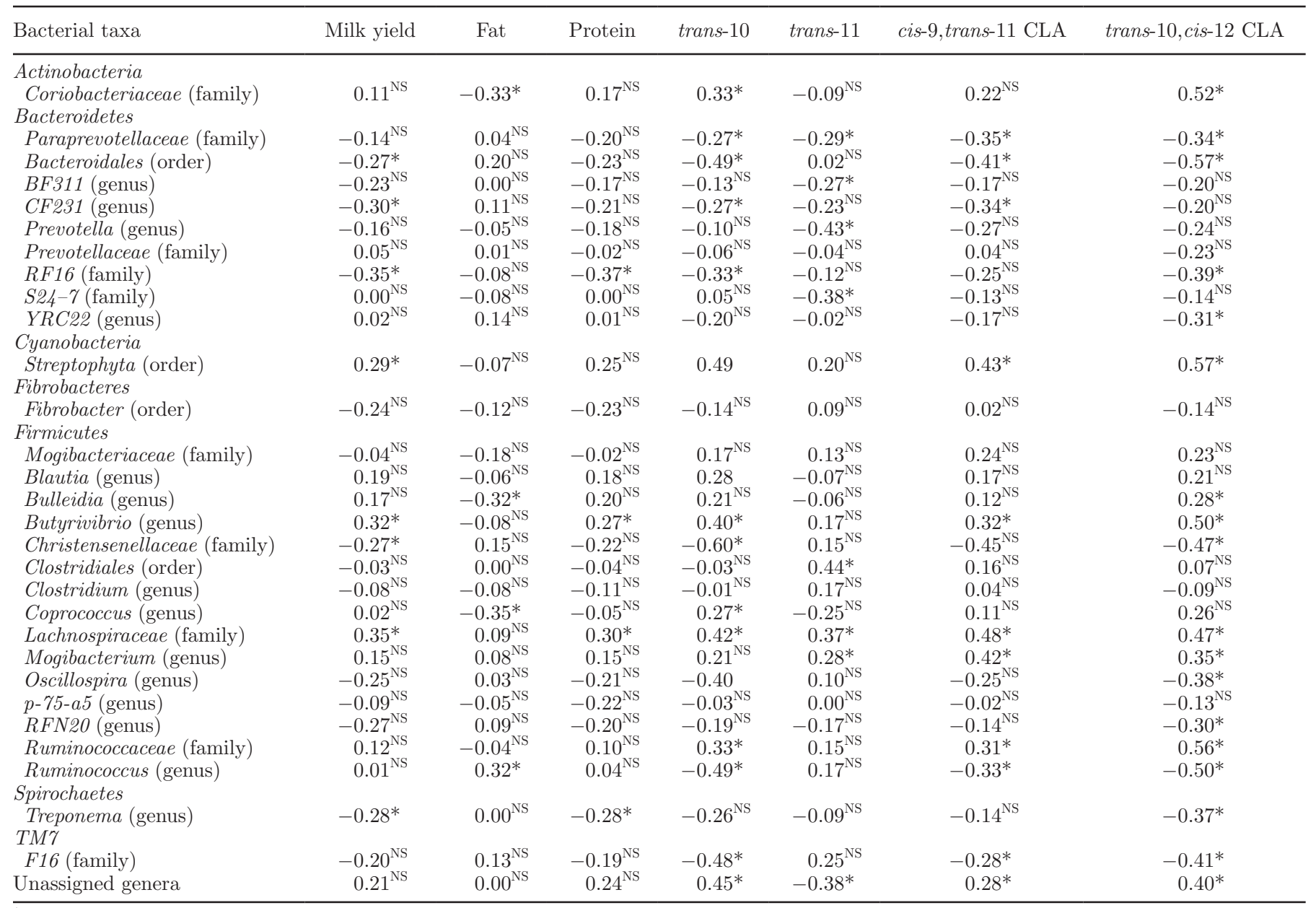

${ }^{1}$ The values in the table shows Pearson's correlation coefficients.

$* P \leq 0.05$.

positive correlation with trans-10,cis-12 CLA and the trans-10 isomer (Table 4).

In the solid fraction, only a few of all the genera showed negative associations with milk yield, protein, and fat concentrations (Table 5). Although most relative abundance of Bacteroidetes lineages showed a negative correlation trend with the FA isomers, only a handful reached significance. In contrast, in the Firmicutes phylum, Bulleidia, Butyrivibrio, and Lachnospiraceae showed a positive correlation whereas Christensenellaceae, Clostridium, and Ruminococcus showed negative correlation with trans-10,cis-2 CLA and the trans-10 isomer. Fibrobacter showed a negative correlation with trans-10,cis-12 CLA and the trans-10 isomer.

\section{DISCUSSION}

Dietary lipids constitute about 4 to $6 \%$ of dietary DM in dairy cow rations (Naik, 2013; Rico and Harvatine,
2013). During microbial fermentation in the rumen, PUFA undergo BH to form SFA that are less toxic to microbes (Lourenço et al., 2010). Under normal BH reactions, the most predominant bioactive FA found in the milk is cis-9,trans-11 CLA (Bauman et al., 2000); however, increasing the PUFA content and fermentability of diets can trigger alternate $\mathrm{BH}$ pathways, leading to the formation of trans-10,cis-12 CLA and other trans FA in the rumen (Rico et al., 2014). Trans-10, cis-12 CLA and other bioactive FA, upon reaching the mammary gland, inhibit fatty acid synthesis leading to milk fat depression in dairy cows (Harvatine et al., 2009). Although MFD is the consequence of microbial action, not all of the rumen microbes participating in normal and alternate BH pathways are known (Rico et al., 2015b). In our study, using the archived rumen samples from an induction and recovery experiment of MFD from Rico et al. (2014), changes in relative abundance of ruminal bacterial populations associated with normal 
Table 5. Association between relative sequence abundance of bacterial taxa and production responses in the solid fraction ${ }^{1}$

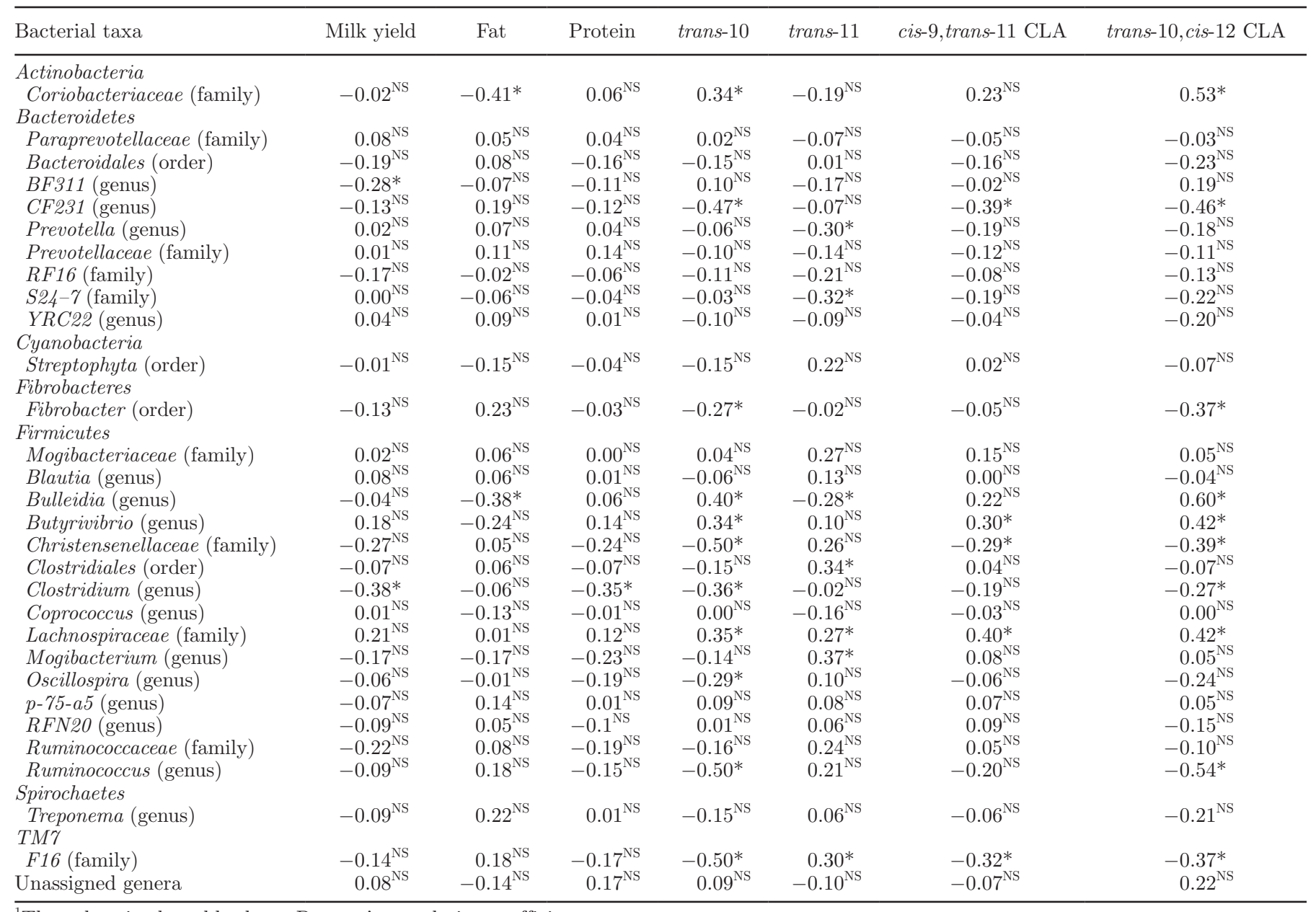

${ }^{1}$ The values in the table shows Pearson's correlation coefficients.

$* P \leq 0.05$.

and alternate $\mathrm{BH}$ pathways were explored. Because the study employed next-generation sequencing technology, which provides an indirect and semiquantitative estimate of the ruminal bacterial populations, the correlation analysis between the identified ruminal bacterial populations and FA isomers detected in milk is speculative. Nevertheless, these predictions may enable linking dietary nutrients, microbiota, and production parameters to gain insights into the role of ruminal bacteria in normal and altered $\mathrm{BH}$ patterns.

Many different nutritional and non-nutritional risk factors can lead to MFD in dairy cows (Jenkins and Harvatine, 2014). Dietary components, particularly altering NDF and starch concentrations in the diet, have an effect on rumen microbiota by modifying the rumen environment (Lourenço et al., 2010). Rico et al. (2015a) described that a drop in $\mathrm{pH}$ was associated with an increase in dietary starch concentration, which can alter the microbial composition leading to significant changes in BH pathways (Fuentes et al., 2009). In our study, higher concentrations of starch and PUFA in the induction diet were expected to have led to changes in the ruminal environment, resulting in alterations of the ruminal bacterial composition. The current experiment modified both starch and PUFA level, and it is impossible to attribute responses to each individually. However, higher concentrations of PUFA, particularly linoleic acid, are known to negatively affect the growth of numerous species of ruminal bacteria (Maia et al., 2010; Huws et al., 2015), and correlation analysis revealed associations between FA isomers and ruminal bacteria in the current study. Dietary starch levels modify the rumen environment and microbial population, but PUFA modify the microbial population and increase the amount of UFA for BH. In support of this, Rico et al. (2015b) observed that correction of dietary starch, but not PUFA, resulted in an inhibition of recovery from MFD. The interaction of starch and PUFA 
level and rumen inoculation on microbial changes associated with MFD will require additional experiments.

According to Rico et al. (2014), signs of MFD were noted by d 10 of the induction period. This was typified by a reduction in milk fat yield of $29 \%$ and milk fat concentration of $30 \%$, without altering milk yield and milk protein yield and concentration. Congruent to changes in milk FA profiles reported in Rico et al. (2014), rumen bacterial profiles on d 10 observed in the present study were found to be significantly different from those of the recovery period. The number of bacterial populations (species richness) and their distribution (diversity) were remarkably reduced on $\mathrm{d}$ 10, similar to the findings of Huws et al. (2015), who reported a general reduction in the abundance of several ruminal bacterial populations in steers fed different oil supplements enriched in PUFA. Lower species richness and diversity are often associated with an altered state in the microbiota, particularly when opportunistic bacteria replace commensal bacteria in the gut, leading to perturbations in the gut microbiome (Fecteau et al., 2016). It was evident from our study that dietary modifications significantly altered the bacterial community composition, as revealed by principal coordinate analysis, suggesting that MFD observed on d 10 is associated with an altered state in the rumen microbiome.

At d 10, the relative abundance of lineages from Firmicutes and Actinobacteria were higher at the expense of Bacteroidetes in both ruminal fractions compared with d 28. This finding, along with the negative correlation between Bacteroidetes members and FA isomers noted in our study, suggest that elevated concentrations of dietary PUFA and starch may exert inhibitory effects on Bacteroidetes. Interestingly, Jami et al. (2014) reported the ratio of Firmicutes to Bacteroidetes is positively correlated with milk fat percentage. Further, a decrease in gut Bacteroidetes indicated an increase in fat concentrations in blood and tissues (Turnbaugh et al., 2006). These reports support the current observation that dietary PUFA and ruminal Bacteroidetes are negatively correlated. Relative abundance of the genus Prevotella constituted about 35\% of Bacteroidetes in our study. This genus, when present in reduced abundances, was associated with an increase in relative abundance of members of Firmicutes, such as Bulleidia and Lachnospiraceae (Jami et al., 2014), similar to findings from the current study. In our study, the relative abundance of Bacteroidetes members in the liquid fraction appear more sensitive to increasing PUFA and starch than its counterparts in the solid fraction, wherein abundant genera including Prevotella, Bacteroidales, and S24-7 were substantially lower on d 10. According to Rico et al. (2015b), Prevotella ruminicola was more sensitive to higher PUFA and starch concentrations whereas
Prevotella bryantii remained unaltered. Although the exact species of Prevotella could not be determined in our study, uncultured Prevotella, which is a major contributor to the rumen microbiota (Pitta et al., 2010, 2014a), was reduced at MFD.

Earlier reports indicated that several bacterial populations, including Prevotella, unclassified genera of Bacteroidales, Lachnospiraceae, Clostridiaceae, and Ruminococcaceae, have the potential to participate in BH reactions (Kim et al., 2008; Belenguer et al., 2010; Huws et al., 2011). However, these findings were contrasted by a more recent report by Huws et al. (2015), who revealed that feeding different oil supplements, such as flaxseed and echium oil, had a negative effect on most bacterial populations in the rumen of steers maintained on grass silage diets, with only a few members of Firmicutes resisting the toxic effects of PUFA. These observations imply that despite the different source of oil supplements used, higher concentrations of PUFA can be detrimental to Bacteroidetes. However, it is not understood why these populations change with increasing doses of dietary PUFA or what their interactions are with other dietary nutrients. Future studies should aim to investigate the effect of increasing doses of dietary PUFA and starch on specific bacterial populations in Bacteroidetes and Firmicutes in a temporal manner, such that dietary recommendations can be developed to bypass the toxic effects of dietary PUFA.

The relative abundance of Firmicutes members, particularly lineages from unclassified Lachnospiraceae, Butyrivibrio, and Bulleidia, were higher on d 10. Among the cultured Firmicutes representatives known to participate in $\mathrm{BH}$, the cultivated strains Butyrivibrio fibrisolvens and the Pseudobutyrivibrio clade are the most active (Lourenço et al., 2010). Two species of Butyrivibrio (i.e., B. fibrisolvens and Butyrivibrio hungatei) had different responses when exposed to the induction diet, as described by Rico et al. (2015b). Those authors reported that $B$. fibrisolvens decreased whereas $B$. hungatei remained unaltered over a $10-\mathrm{d}$ induction period. Although individual species of Butyrivibrio were not identified in our study, the relative abundance of this genus was higher on d 10, showing its resistance to higher concentrations of PUFA and starch. Lourenço et al. (2010) reported increased resistance of Butyrivibrio to PUFA. Interestingly, this genus along with Lachnospiraceae and Bulleidia showed positive correlations with all FA isomers. Although the current study revealed that more uncultivated bacteria, in addition to Butyrivibrio, resist the higher concentrations of PUFA and starch and are positively associated with FA isomers, their role in $\mathrm{BH}$ is yet to be determined. More in-depth analysis combined with metagenomic and metatranscriptomic approaches when applied to such 
induction and recovery models of MFD may provide insights on the functional role of these bacterial genera in $\mathrm{BH}$.

Using flaxseed and echium oils supplemented to steers, Huws et al. (2015) demonstrated that Butyrivibrio, Howardella, Oribacterium, Pseudobutyrivibrio, and Roseburia were increased with flaxseed oil, whereas Succinivibrio and Roseburia were the only genera that increased with echium oil. In our study, soybean oil was used as a PUFA substrate, which may be different in composition compared with the PUFA source used in other studies (Huws et al., 2011, 2015). Differences in PUFA source may have partially contributed to differences in the relative abundance of bacterial populations between the current study and Huws et al. (2011, 2015). These findings coincide with the observation that, depending on the substrate used for BH, effects on bacterial populations differed (Patra and Yu, 2012).

Coriobacteriaceae, a family of Actinobacteria, was abundant on d 10. Similar to unclassified Lachnospiraceae, Butyrivibrio, and Bulleidia, this genus was positively correlated with trans-10,cis-12 CLA and trans-10 isomers. However, this lineage does not show any association with cis-9,trans-11 CLA, suggesting its potential role in altered $\mathrm{BH}$ pathways. Coriobacteriaceae has been implicated with dyslipidemic phenotypes including obesity, metabolic syndrome, and hypercholesterolemia (Martínez et al., 2013). Further, these authors reported this bacterial family has also been suggested as a microbial target to regulate cholesterol metabolism. Zened et al. (2011) illustrated that Coriobacteriaceae is positively associated with the production of trans-10 isomers, but indicated further studies are required to establish the correlation.

We concluded that alterations in milk FA isomers caused by elevated PUFA and starch are associated with alterations in microbiota and the current study revealed the positive association between species of Bulleidia, Butyrivibrio, Lachnospiraceae, and Coriobacteriaceae and diet-induced MFD. However, the functional role of these uncultivated bacteria in normal and altered $\mathrm{BH}$ pathways is yet to be determined. Fibrobacter, a gram-negative bacterium, is described as a dominant cellulolytic bacterial genus of the rumen (Amann et al., 1992; Suen et al., 2011) with Fibrobacter succinogenes S85 as the type strain. Sensitivity of F. succinogenes to higher concentrations of starch and PUFA was demonstrated as early as d 3, when dairy cows were exposed to low-fiber, high-starch, high-PUFA diets (Rico et al., 2015a). Similar findings were noted in our study, where the relative abundance of Fibrobacter was lower on d 10 in the solid fraction compared with the recovery period samples. Fibrobacter showed negative correlation with trans-10,cis-12 CLA and trans-10 FA isomers. Due to the cellulolytic nature of Fibrobacter, these associations may have been driven by increased starch and consequently lowered $\mathrm{pH}$ in the rumen environment (Sung et al., 2007), and these alterations might have been restored with the increased concentration of NDF in the recovery diet.

Our study is the first to investigate how alterations in ruminal bacteria gradually reverted from a MFD condition to a normal state. The bacterial communities on d 28 clustered with those of the donor inoculum samples, revealing the d- 28 bacterial communities have been restored to a typical non-MFD healthy state from the perturbed condition on d 10. This implies that, to achieve a normal pattern of milk profiles (reduction in trans-10,cis-12 CLA and trans-10 isomer and rebalancing of cis-9,trans-11 CLA) from MFD, a period of up to $18 \mathrm{~d}$ is required for microbiota to adjust and adapt to the switch to the recovery diet, which agrees with the general observation that microbiota need about 2 wk to acclimatize to a new diet (Anderson et al., 2016; Machado et al., 2016).

The most obvious change observed with increased NDF and reduced starch and PUFA concentrations in the diet was an increase in the relative abundance of Bacteroidetes lineages and a decrease in Firmicutes members. These effects spiked on d 13 and gradually stabilized by d 28 (Figure 3). Several genera, including Prevotella, S24-7, Clostridiales, Mogibacterium, and F16, showed fluctuations between d 13 and 16 and $\mathrm{d} 13$ and 28. These differences were not observed between d 16 and 28, probably indicating adaptation to the recovery diet. Although it was not possible to explain the biphasic pattern of milk FA profiles from a microbial perspective during the recovery period, it can be concluded that, with a dietary shift to higher NDF concentrations, bacterial populations experienced fluctuations in the first few days but appear to stabilize by $\mathrm{d} 28$.

Evidence indicates a strong host-microbiota specificity in ruminants (Kittelmann et al., 2014; Weimer, 2015; Weimer et al., 2015); thus, any changes induced in the rumen microbiota as a result of rumen digesta exchange or treatment with pro- or prebiotics, enzymes, or chemical compounds would cause the microbial community composition to revert to the original pretreatment composition once ceased (Weimer et al., 2010). In our study, inoculation with 10\% (approximately $8 \mathrm{~kg}$ ) rumen digesta from non-MFD cows to MFD recipient cows showed subtle changes in the ruminal bacteria relative to the changes induced by the dietary switch. The inoculation procedure was performed for 6 consecutive days (d 11-17), which resulted in a slightly accelerated recovery by d 13 compared with dietary intervention alone (Rico et al., 2014). Inoculation did not appear to 
alter the bacterial community in its entirety, but had small specific changes, particularly on d 16 of sampling. We did not investigate changes on the subsequent days following d 16 to 28; therefore, it is not known how long these changes were persistent. However, changes observed on d 16, which appear to be driven by inoculation, disappeared by d 28, indicating transient effects, which concurs with Weimer et al. (2010).

In conclusion, diet-induced MFD in dairy cows was accompanied by significant shifts in the relative abundance of the ruminal bacterial populations. Dietinduced MFD showed a negative correlation with the relative abundance of some bacteria, including members of Bacteroidetes and Fibrobacteres. In contrast, the relative abundance of specific genus level lineages of Firmicutes and Actinobacteria, such as unclassified Lachnospiraceae, Butyrivibrio, and Bulleidia and unclassified Coriobacteriaceae, respectively, were increased in diet-induced MFD dairy cows. These genera also showed positive correlation with trans-10, cis-12 CLA and trans-10 isomers, suggesting their potential role in altered $\mathrm{BH}$ pathways. Inoculation of rumen contents (10\%) from non-MFD cows to diet-induced MFD recipient cows did not induce wholesale shifts in the microbiota, but affected a few specific bacteria only on $\mathrm{d}$ 16. More in-depth studies using advanced metagenomic and metatranscriptomic approaches are required to understand the effect of both starch and PUFA on rumen bacteria and the role of uncultivated rumen bacteria in normal and altered BH pathways. Such studies will help design nutritional and non-nutritional strategies to prevent microbial perturbations in the rumen environment.

\section{ACKNOWLEDGMENTS}

We are grateful to the Biomedical Research Core Facilities, University of Pennsylvania, for sequencing services.

\section{REFERENCES}

Amann, R. I., C. Lin, R. Key, L. Montgomery, and D. A. Stahl. 1992. Diversity among Fibrobacter isolates: Towards a phylogenetic classification. Syst. Appl. Microbiol. 15:23-31.

Anderson, C. L., C. Schneider, G. Erickson, J. MacDonald, and S. C. Fernando. 2016. Rumen bacterial communities can be acclimated faster to high concentrate diets than currently implemented feedlot programs. J. Appl. Microbiol. 120:588-599.

Anderson, M. J. 2001. A new method for non-parametric multivariate analysis of variance. Austral Ecol. 26:32-46.

Bauman, D., L. Baumgard, B. Corl, and d. J. Griinari. 2000. Biosynthesis of conjugated linoleic acid in ruminants. J. Anim. Sci. 77(E-Suppl.):1-15.

Bauman, D. E., and J. M. Griinari. 2003. Nutritional regulation of milk fat synthesis. Annu. Rev. Nutr. 23:203-227.
Belenguer, A., P. G. Toral, P. Frutos, and G. Hervás. 2010. Changes in the rumen bacterial community in response to sunflower oil and fish oil supplements in the diet of dairy sheep. J. Dairy Sci. 93:3275-3286.

Caporaso, J. G., K. Bittinger, F. D. Bushman, T. Z. DeSantis, G. L. Andersen, and R. Knight. 2010b. PyNAST: A flexible tool for aligning sequences to a template alignment. Bioinformatics $26: 266-267$.

Caporaso, J. G., J. Kuczynski, J. Stombaugh, K. Bittinger, F. D. Bushman, E. K. Costello, N. Fierer, A. G. Pena, J. K. Goodrich, and J. I. Gordon. 2010a. QIIME allows analysis of high-throughput community sequencing data. Nat. Methods 7:335-336.

Dollive, S., G. L. Peterfreund, S. Sherrill-Mix, K. Bittinger, R. Sinha, C. Hoffmann, C. S. Nabel, D. A. Hill, D. Artis, and M. A. Bachman. 2012. A tool kit for quantifying eukaryotic rRNA gene sequences from human microbiome samples. Genome Biol. 13:R60.

Edgar, R. C. 2010. Search and clustering orders of magnitude faster than BLAST. Bioinformatics 26:2460-2461.

Fecteau, M.-E., D. W. Pitta, B. Vecchiarelli, N. Indugu, S. Kumar, S. C. Gallagher, T. L. Fyock, and R. W. Sweeney. 2016. Dysbiosis of the fecal microbiota in cattle infected with Mycobacterium avium ssp. paratuberculosis. PLoS One 11:e0160353.

Fuentes, M. C., S. Calsamiglia, P. Cardozo, and B. Vlaeminck. 2009. Effect of $\mathrm{pH}$ and level of concentrate in the diet on the production of biohydrogenation intermediates in a dual-flow continuous culture. J. Dairy Sci. 92:4456-4466.

Garton, G. A. 1977. Fatty acid metabolism in ruminants. Page 337370 in Biochemistry of Lipids II. T. W. Goodwin, ed. Univ. Park Press, Baltimore, MD.

Harvatine, K. J., Y. Boisclair, and D. Bauman. 2009. Recent advances in the regulation of milk fat synthesis. Animal 3:40-54.

Huws, S. A., E. J. Kim, S. J. Cameron, S. E. Girdwood, L. Davies, J. Tweed, H. Vallin, and N. D. Scollan. 2015. Characterization of the rumen lipidome and microbiome of steers fed a diet supplemented with flax and echium oil. Microb. Biotechnol. 8:331-341.

Huws, S. A., E. J. Kim, M. R. Lee, M. B. Scott, J. K. Tweed, E. Pinloche, R. J. Wallace, and N. D. Scollan. 2011. As yet uncultured bacteria phylogenetically classified as Prevotella, Lachnospiraceae incertae sedis and unclassified Bacteroidales, Clostridiales and Ruminococcaceae may play a predominant role in ruminal biohydrogenation. Environ. Microbiol. 13:1500-1512.

Indugu, N., K. Bittinger, S. Kumar, B. Vecchiarelli, and D. Pitta. 2016. A comparison of rumen microbial profiles in dairy cows as retrieved by 454 Roche and Ion Torrent (PGM) sequencing platforms. PeerJ 4:e1599.

Jami, E., B. A. White, and I. Mizrahi. 2014. Potential role of the bovine rumen microbiome in modulating milk composition and feed efficiency. PLoS One 9:e85423.

Jenkins, T. C., R. Wallace, P. Moate, and E. Mosley. 2008. Boardinvited review: Recent advances in biohydrogenation of unsaturated fatty acids within the rumen microbial ecosystem. J. Anim. Sci. 86:397-412.

Jenkins, T. C., and K. J. Harvatine. 2014. Lipid feeding and milk fat depression. Vet. Clin. North Am. Food Anim. Pract. 30:623-642.

Kim, E. J., S. A. Huws, M. R. Lee, J. D. Wood, S. M. Muetzel, R. J. Wallace, and N. D. Scollan. 2008. Fish oil increases the duodenal flow of long chain polyunsaturated fatty acids and trans-11 18:1 and decreases 18:0 in steers via changes in the rumen bacterial community. J. Nutr. 138:889-896.

Kim, Y. J., R. Liu, J. Rychlik, and J. Russell. 2002. The enrichment of a ruminal bacterium (Megasphaera elsdenii YJ-4) that produces the trans-10, cis-12 isomer of conjugated linoleic acid. J. Appl. Microbiol. 92:976-982.

Kittelmann, S., C. S. Pinares-Patiño, H. Seedorf, M. R. Kirk, S. Ganesh, J. C. McEwan, and P. H. Janssen. 2014. Two different bacterial community types are linked with the low-methane emission trait in sheep. PLoS One 9:e103171.

Lourenço, M., E. Ramos-Morales, and R. Wallace. 2010. The role of microbes in rumen lipolysis and biohydrogenation and their manipulation. Animal 4:1008-1023. 
Lozupone, C., and R. Knight. 2005. UniFrac: A new phylogenetic method for comparing microbial communities. Appl. Environ. Microbiol. 71:8228-8235.

Machado, M. G., E. Detmann, H. C. Mantovani, S. C. Valadares Filho, C. B. Bento, M. I. Marcondes, and A. S. Assunção. 2016. Evaluation of the length of adaptation period for changeover and crossover nutritional experiments with cattle fed tropical forage-based diets. Anim. Feed Sci. Technol. 222:132-148.

Maia, M. R., L. C. Chaudhary, C. S. Bestwick, A. J. Richardson, N. McKain, T. R. Larson, I. A. Graham, and R. J. Wallace. 2010. Toxicity of unsaturated fatty acids to the biohydrogenating ruminal bacterium, Butyrivibrio fibrisolvens. BMC Microbiol. 10:52.

Martínez, I., D. J. Perdicaro, A. W. Brown, S. Hammons, T. J. Carden, T. P. Carr, K. M. Eskridge, and J. Walter. 2013. Dietinduced alterations of host cholesterol metabolism are likely to affect the gut microbiota composition in hamsters. Appl. Environ. Microbiol. 79:516-524.

McDonald, D., M. N. Price, J. Goodrich, E. P. Nawrocki, T. Z. DeSantis, A. Probst, G. L. Andersen, R. Knight, and P. Hugenholtz. 2012. An improved Greengenes taxonomy with explicit ranks for ecological and evolutionary analyses of bacteria and archaea. ISME J. 6:610-618.

McKenna, P., C. Hoffmann, N. Minkah, P. P. Aye, A. Lackner, Z. Liu, C. A. Lozupone, M. Hamady, R. Knight, and F. D. Bushman. 2008. The macaque gut microbiome in health, lentiviral infection, and chronic enterocolitis. PLoS Pathog. 4:e20.

Morgavi, D. P., W. Kelly, P. Janssen, and G. Attwood. 2013. Rumen microbial (meta) genomics and its application to ruminant production. Animal 7(Suppl. 1):184-201.

Naik, P. 2013. Bypass fat in dairy ration-A review. Anim. Nutr. Feed Technol. 13:147-163.

Patra, A. K., and Z. Yu. 2012. Effects of essential oils on methane production and fermentation by, and abundance and diversity of, rumen microbial populations. Appl. Environ. Microbiol. 78:42714280.

Pitta, D. W., S. Kumar, B. Vecchiarelli, D. Shirley, K. Bittinger, L. Baker, J. Ferguson, and N. Thomsen. 2014a. Temporal dynamics in the ruminal microbiome of dairy cows during the transition period. J. Anim. Sci. 92:4014-4022.

Pitta, D. W., W. Pinchak, S. Dowd, K. Dorton, I. Yoon, B. Min, J. Fulford, T. Wickersham, and D. Malinowski. 2014c. Longitudinal shifts in bacterial diversity and fermentation pattern in the rumen of steers grazing wheat pasture. Anaerobe 30:11-17.

Pitta, D. W., N. Parmar, A. K. Patel, N. Indugu, S. Kumar, K. B. Prajapathi, A. B. Patel, B. Reddy, and C. Joshi. 2014d. Bacterial diversity dynamics associated with different diets and different primer pairs in the rumen of Kankrej cattle. PLoS One 9:e111710.

Pitta, D. W., W. E. Pinchak, S. E. Dowd, J. Osterstock, V. Gontcharova, E. Youn, K. Dorton, I. Yoon, B. R. Min, and J. Fulford. 2010. Rumen bacterial diversity dynamics associated with changing from bermudagrass hay to grazed winter wheat diets. Microb. Ecol. 59:511-522.

Price, M. N., P. S. Dehal, and A. P. Arkin. 2010. FastTree 2-Approximately maximum-likelihood trees for large alignments. PLoS One 5:e9490.

R Core Team. 2014. R: A language and environment for statistical computing. R Foundation for Statistical Computing, Vienna, Austria.
Rico, D. E., A. Holloway, and K. Harvatine. 2015b. Effect of diet fermentability and unsaturated fatty acid concentration on recovery from diet-induced milk fat depression. J. Dairy Sci. 98:7930-7943.

Rico, D. E., S. Preston, J. Risser, and K. Harvatine. 2015a. Rapid changes in key ruminal microbial populations during the induction of and recovery from diet-induced milk fat depression in dairy cows. Br. J. Nutr. 114:358-367.

Rico, D. E., Y. Ying, A. Clarke, and K. Harvatine. 2014. The effect of rumen digesta inoculation on the time course of recovery from classical diet-induced milk fat depression in dairy cows. J. Dairy Sci. 97:3752-3760.

Rico, D. E., and K. J. Harvatine. 2013. Induction of and recovery from milk fat depression occurs progressively in dairy cows switched between diets that differ in fiber and oil concentration. J. Dairy Sci. 96:6621-6630.

Salipante, S. J., D. J. Sengupta, C. Rosenthal, G. Costa, J. Spangler, E. H. Sims, M. A. Jacobs, S. I. Miller, D. R. Hoogestraat, and B. T. Cookson. 2013. Rapid 16S rRNA next-generation sequencing of polymicrobial clinical samples for diagnosis of complex bacterial infections. PLoS One 8:e65226.

Suen, G., P. J. Weimer, D. M. Stevenson, F. O. Aylward, J. Boyum, J. Deneke, C. Drinkwater, N. N. Ivanova, N. Mikhailova, and O. Chertkov. 2011. The complete genome sequence of Fibrobacter succinogenes S85 reveals a cellulolytic and metabolic specialist. PLoS One 6:e18814.

Sung, H. G., Y. Kobayashi, J. Chang, A. Ha, I. H. Hwang, and J. K. Ha. 2007. Low ruminal $\mathrm{pH}$ reduces dietary fiber digestion via reduced microbial attachment. Asian-Australas. J. Anim. Sci. 20:200.

Turnbaugh, P. J., R. E. Ley, M. A. Mahowald, V. Magrini, E. R. Mardis, and J. I. Gordon. 2006. An obesity-associated gut microbiome with increased capacity for energy harvest. Nature 444:1027-1131.

Wang, Q., G. M. Garrity, J. M. Tiedje, and J. R. Cole. 2007. Naive Bayesian classifier for rapid assignment of rRNA sequences into the new bacterial taxonomy. Appl. Environ. Microbiol. 73:5261-5267.

Weimer, P. J., L. D. S. Cabral, and F. Cacite. 2015. Effects of ruminal dosing of Holstein cows with Megasphaera elsdenii on milk fat production, ruminal chemistry, and bacterial strain persistence. J. Dairy Sci. 98:8078-8092.

Weimer, P. J., D. Stevenson, H. Mantovani, and S. Man. 2010. Host specificity of the ruminal bacterial community in the dairy cow following near-total exchange of ruminal contents. J. Dairy Sci. 93:5902-5912.

Weimer, P. J. 2015. Redundancy, resilience, and host specificity of the ruminal microbiota: Implications for engineering improved ruminal fermentations. Front. Microbiol. 6:296

Wu, G. D., J. D. Lewis, C. Hoffmann, Y.-Y. Chen, R. Knight, K. Bittinger, J. Hwang, J. Chen, R. Berkowsky, and L. Nessel. 2010. Sampling and pyrosequencing methods for characterizing bacterial communities in the human gut using $16 \mathrm{~S}$ sequence tags. $\mathrm{BMC}$ Microbiol. 10:206.

Zened, A., S. Combes, L. Cauquil, C. Rousseau, C. Klopp, A. Troegeler-Meynadier, and F. Enjalbert. 2011. The ruminal level of trans-10 fatty acids of dairy cows is linked to the composition of bacterial community. In 4th Congress of European Microbiologists, Geneva, Switzerland. 